\title{
Cantor's Power-Set Theorem Versus Frege's Double-Correlation Thesis
}

\author{
Nino B. Cocchiarella
}

\begin{abstract}
Frege's thesis that second-level concepts can be correlated with firstlevel concepts and that the latter can be correlated with their value-ranges is in direct conflict with Cantor's power-set theorem, which is a necessary part of the iterative, but not of the logical, concept of class. Two consistent second-order logics with nominalized predicates as abstract singular terms are described in which Frege's thesis and the logical notion of a class are defended and Cantor's theorem is rejected. Cantor's theorem is not incompatible with the logical notion of class, however. Two alternative similar kinds of logics are also described in which Cantor's theorem and the logical notion of a class are retained and Frege's thesis is rejected.
\end{abstract}

\section{Cantor's Power-Set Theorem and the Logical Concept of Class}

It is taken as an obvious "truism" today that Cantor's power-set theorem together with the assumption that there is a universal class implies Cantor's paradox (cf. Quine 1963, 202). For, by definition of what it means for one class to have a greater cardinal number of members than another, Cantor's theorem that any function (many-one relation) from a class into the class of all subclasses of that class cannot be onto the latter - i.e., that at least one subclass of that class is not in the range of the function - has the consequence that the cardinal number of the class of all subclasses of a class is always greater than the cardinal number of that class, and therefore that there can be no greatest cardinal number. The universal class, however, contains every class, including the class of all its subclasses, and therefore no class can have a greater cardinal number of members than the universal class, i.e., the universal class has the greatest cardinal number, from which it follows that there is a greatest cardinal number after all. ${ }^{1}$

\footnotetext{
${ }^{1}$ For the sake of historical accuracy I should note that I. Grauan-Guiness informs me that Cantor's paradox is the claim that there is a greatest cardinal number that is both identical with itself and greater than itself, and that the way Cantor arrived at this "paradox" probably had nothing to do with his power-set theorem and indeed predates it. The version we give here is the way Cantor's paradox is usually understood today.
} 
The assumption that there is a universal class is an essential part of what is known as the logical notion of a class, i.e., the notion of a class as the extension of a concept. Cantor's theorem, on the other hand, is an essential part of the mathematical notion of a class, i.e., the notion of a class as a set, which is also called the iterative concept of set (Boolos 1971). As based on the iterative concept, classes as sets are formed in stages by iterating the operation: $X \rightarrow$ $X \cup \mathcal{P}(X)$, where $\mathcal{P}(X)$ is the power set of $X$, i.e., the set of all subsets of $X$, which, by Cantor's theorem, has a greater cardinal number of members than $X$ has. The stages in which classes as sets are formed in this way are assumed to be well-ordered so that each stage corresponds to some ordinal number (up to, at least, some limit ordinal, if the stages are assumed to terminate at all). It is for this reason that this notion of class is said to be a mathematical, and not a logical, notion.

The mathematical and the logical notions of a class can be combined, but only by seriously restricting the logical notion so that not all classes in the logical sense are sets (or have sets corresponding to them). Thus, given, as essential to the iterative concept of set as based on Cantor's theorem, the power-set axiom that the class of all subsets of any set is itself a set, it follows by Cantors theorem that the universal class is not a set. ${ }^{2}$ In such a combined framework, classes that are not sets (or that do not have sets corresponding to them) are called "ultimate", or "proper", classes, and, it is generally assumed, that while such classes have members, they cannot themselves be members, whether of sets or of ultimate classes. Thus, for example, although every set is a member of the universal class in such a framework, the universal class cannot be a member of itself, nor of any other class (not even its singleton, or other finite classes). It is through such a restriction on classes in the logical sense that Cantor's paradox is avoided; for, in such a framework, Cantor's theorem is assumed to apply only to classes that are sets.

The restriction, if not total elimination, of classes in the logical sense is usually said to be necessary not because of Cantor's paradox but because of Russell's paradox. Russell's paradox, however, as Russell himself made clear, was based on Cantor's theorem and the assumption that there is a universal class. Thus, Russell claimed that he was led to his paradox "in the endeavour to reconcile Cantor's proof that there can be no greatest cardinal number with the very plausible supposition that the class of all terms (which we have seen to be essential to all formal propositions) has necessarily the greatest possible number of members" (Russell 1903, 101). Russell then described the derivation of his paradox through the construction of a certain many-one relation defined

${ }^{2}$ The argument, succinctly stated as follows, is easily seen to be valid:

The cardinal number of the class of all subsets of any set is greater than the cardinal number of that set (Cantor's theorem). The class of all subsets of any set is itself a set (power-set axiom). The cardinal number of any set is not greater than the cardinal number of any set containing that set (by definition of greater cardinality). The universal class (by definition) contains every class. Therefore, the universal class is not a set. 
over the universal class as follows (p. 367):

In the case of terms and classes, if $x$ be not a class, let us correlate it with $\iota x$, i.e., the class whose only member is $x$, but if $x$ be a class, let us correlate it with itself. (This is not a one-one, but a many-one correlation, for $x$ and $\iota x$ are both correlated with $\iota x$. . .) Then the class which, according to Cantor's argument, should be omitted from the correlation, is the class $w$ of those classes which are not members of themselves; yet this, being a class, should be correlated with itself.

Here we have a clear application of Cantor's theorem that any many-one relation from a class into the. class of all subclasses of that class cannot be onto the latter, i.e., that at least one subclass of that class is not in the range of that relation. As applied to the universal class and the many-one relation in question, the subclass that must be omitted is the class of those classes that are not members of themselves, which, as Russell observed, stands to itself in the relation in question (namely, the identity relation as applied to classes) and therefore is in the range of the relation after all. Thus Russell's paradox is but another variation of the conflict between Cantor's theorem and the assumption that there is a universal class.

It does not follow that it is the logical notion of a class, and in particular the assumption that there is a universal class, that must be given up as a result of this conflict with Cantor's theorem. Russell himself, in fact, after discovering his paradox, was by no means ready to give up the logical notion. Thus, although he agreed that "one would be tempted to say that the conception of the totality of things, or of the whole universe of entities and existents, is in some way illegitimate and inherently contrary to logic," nevertheless, he went on to insist that "it is undesirable to adopt so desperate a measure as long as hope remains of some less heroic solution" (1903, 362).

Unfortunately, the "less heroic solution" that Russell adopted in the end, namely, a theory of logical types, is not really philosophically satisfying. For, although the logical notion of a class is retained in such a framework, it is retained, as it were, with a vengeance. In particular, rather than there being just one universal class, there is instead a universal class for each logical type. The logical notion of a class is preserved on this approach, but only at the expense of having it relativised to each of the different logical types.

Of course, strictly speaking, there are no classes in Russell's theory of types, because for him all talk of classes was reducible to talk of concepts, or what he called propositional functions, as higher-order intensional objects. This is not an objection that we will be concerned with here. For the fact that all talk of classes in the logical sense can be reduced and explained in terms of intensional objects does not mean that the logical notion of a class is being rejected or eliminated. That is, the logical notion of a class is just the notion of a class as the extension of a concept, which, as Frege pointed out, is another way of saying that classes in the logical sense have their being in the concepts whose extensions they are 
and not in their members (cf. Frege 1979, 183). We should not be surprised, accordingly, if this notion is somehow reducible, or otherwise explainable, in terns of other more fundamental notions having to do with concepts. For that reason we will not be concerned here with the question of whether the notion of a class in the logical sense is being taken as a primitive or not, i.e., with whether classes and membership are taken as primitives of a logical framework or are analyzed instead in terms of concepts and predication.

There. is another problem with Russell's solution, however, in addition to that of the relativisation of classes to each logical type. This problem has to do with the fact that the particular theory of types that Russell adopted is a theory of ramified types, which, unlike the theory of simple types, is based on a constructive (i.e., "predicative") comprehension principle. Such a constructive approach is not without merit, but it does affect the logical notion of a class in a fundamental way. In particular, because of the kind of constructive constraints imposed by the theory on the comprehension principle, Cantor's theorem, which involves objects of different types, cannot be proved in such a framework (cf. Quine 1963. 265). That is not objectionable in itself, but it does not get at the root of the matter of the real conflict between Cantor's power-set theorem and the logical notion of class as represented by an impredicative comprehension principle.

An impredicative comprehension principle is provable in the theory of simple types. But in this framework, as in the theory of ramified types as well, Russell's paradox cannot even be stated (because of the grammatical constraints on the conditions of well-formedness), which means that the description of the class upon which Russell's paradox is based is meaningless. Thus, not only must the universal class be relativised and duplicated, potentially, infinitely many times in order to avoid Russell's paradox on this approach, but the paradox must itself be ruled as meaningless. The theory of types, whether simple or ramified, is not really a solution of the problem so much as a way of avoiding it altogether.

There is another way in which we can preserve our logical intuitions and not give up the logical notion of a class in favor of the mathematical (i.e., in favor of set theory), and yet in which not only is Cantor's theorem formulable but so is Russell's paradox (though, of course, the latter will no longer be provable). Indeed, there is not just one such way, but at least two (both of which themselves have two alternatives). On the first, it is not the logical notion of a class that must be rejected as the way of resolving Russell's paradox, but Cantor's theorem instead. This rejection is not ad hoc or arbitrary on this approach, but is based on a more general principle, which we refer to as Frege's double-correlation thesis. It is this approach that we will turn to first. On the second and alternative approach, which we will turn to later, the trouble lies in neither Cantor's theorem nor in the assumption that there is a universal class (both of which can be retained without contradiction on this approach), but rather in how the logic of identity is to be applied in certain contexts. On this approach, the claim that a contradiction results by combining Cantor's theorem with the assumption that the universal class exists is not a "truism" after all but is outright false. 


\section{Second-order Logic with Nominalized Predi- cates as Abstract Singular Terms}

Frege was the first to give not only a complete formulation of first-order logic but a formulation of standard second-order logic as well, and it was in the context of the latter that he attempted to present the logical notion of a class (or what he called a Wertverlauf). Unfortunately, it was that presentation that fell to Russell's paradox. In writing Frege of his paradox, Russell described it not only in terms of the class of those classes that are not members of themselves but also in terms of predication (where by a predicate Russell meant a monadic concept): "Let $w$ be the predicate of being predicate which cannot be predicated of itself. Can $w$ be predicated of itself? From either answer follows its contradictory" (Frege 1980, 130). Frege objected to the idea of a concept (or predicate in Russell's terminology) being predicated of (or falling under) itself, and preferred to speak instead of a concept being predicated of its own extension (p. 132f). On his view, concepts have an unsaturated nature, which he explained in terms of their functionality, and it was this unsaturated nature that precluded their being objects or, from a semantical point of view, entities that can be denoted by singular terms. Thus concepts, because they are not objects, cannot be the objects denoted by nominalized predicates as abstract singular terms contrary to what Russell assumed in speaking of "the predicate [concept] of being a predicate that cannot be predicated of itself".

This difference, though fundamental, is not crucial to the way that Russell's paradox applies to Frege's logic. For, even though a nominalized predicate cannot, according to Frege, denote the concept that the predicate otherwise stands for in its role as a predicate, he did agree that it denotes a logical object somehow correlated with that concept, namely, the class (Wertverlauf) that is the extension of that concept. It is only in this way, Frege maintained, that we are able to apprehend classes as logical objects at all. ${ }^{3}$ Thus, Frege's logic of classes is really an extension of standard second-order logic in which nominalized predicates are allowed to occur as abstract singular terms on a par with individual variables and other singular terms, and in particular as singular terms that denote classes (or, more generally, Wertverläufe). It is this sort logical framework - but not necessarily with Frege's view of concepts and the nature of their unsaturatedness - that we will adopt in developing our two approaches toward defending the notion of a class in the logical sense. Instead of Frege's particular view of concepts as functions from objects to truth-values, there is an alternative (which in fact we prefer) in which concepts are viewed as cognitive

\footnotetext{
${ }^{3}$ Cf. Cocchiarella 1987, chapter 2, section 4 . for a detailed justification of the claim that for Frege the objects denoted by nominalized predicates as abstract singular terms are none other than the classes (Wertverläufe) that are the extensions of the concepts that those predicates stand for. In regard to Frege's view of classes as logical objects and our apprehension of them as such. compare Frege's July 1902 letter to Russell: "But the question is. how de we apprehend logical objects? And I have found no other answer to it than this. We apprehend them as extensions of concepts, or more generally, as ranges of values of functions" (Frege 1980, I41).
} 
structures, the unsaturated nature of which is explained in terms of their role as capacities that are exercised in speech acts (and as what informs those acts with predicative content). ${ }^{4}$ We will leave open the question of which particular view to adopt and simply consider the general framework to be "Fregean-like".

Frege did not allow nominalized predicates to occur as such in his extended second-order logic, but used instead the spiritus lenis as an abstraction operator corresponding to the operation of nominalization. That is, instead of allowing $F(F)$ as well-formed, where $F()$ is a monadic predicate and $F$ (without parentheses) is its nominalization. Frege used $F(\grave{x} F(x))$, with $\grave{x} F(x)$ as the way to represent the nominalization of $F()$. We will not represent the operation of nominalization that way here, however, but will simply take the deletion of parentheses (and commas in the case of a relational predicate) as our way of distinguishing (in the object language) the nominalization of a predicate from the predicate itself.

An abstraction operator is useful in the representation of complex predicates, however, and, in conformity with current practice, we will use Church's $\lambda$-operator for that purpose. Thus, where $\varphi\left(x_{1}, \ldots, x_{n}\right)$ is a wff (well-formed formula), $\left[\lambda x_{1} \ldots x_{n} \varphi\left(x_{1}, \ldots, x_{n}\right)\right]($.$) is a complex ( n$-place) predicate, and $\left[\lambda x_{1} \ldots x_{n}\right.$ $\left.\varphi\left(x_{1}, \ldots, x_{n}\right)\right]$ is a complex singular term. (For convenience, we will usually drop the accompanying pair of parentheses in referring to predicates as unsaturated expressions, though whenever a predicate occurs in a wff as a predicate it will always have its accompanying pair of parentheses).

Aside from logical grammar (for the formal details of which see Appendix A), we assume the general framework of standard second-order predicate logic with identity, but extended now to apply to wffs in which nominalized predicates occur as abstract singular terms. The logical laws that apply to $\lambda$-abstracts as complex predicates in this context are (1) $\lambda$-conversion (starred to mark the fact that it also applies to wffs that contain nominalized predicates),

$$
\left[\lambda x_{1} \ldots x_{n} \varphi\right]\left(a_{1}, \ldots, a_{n}\right) \longleftrightarrow \varphi\left(a_{1} / x_{1}, \ldots, a_{n} / x_{n}\right),
$$

where, for $1 \leq i \leq n, a_{i}$ is free for $x_{i}$ in $\varphi$; and (2) the identity law,

$$
\left[\lambda x_{1} \ldots x_{n} F\left(x_{1}, \ldots, x_{n}\right)\right]=F
$$

where $F$ is an $n$-place predicate variable or constant. The (impredicative) comprehension principle of standard second-order predicate logic is also assumed, and, at least initially, we will assume that it is also extended without qualification to apply to wffs that contain nominalized predicates as well (and we use '*' to mark this fact):

$$
\exists F^{n} \forall x_{1} \ldots \forall x_{n}\left(F\left(x_{1}, \ldots, x_{n}\right) \longleftrightarrow \varphi\right)
$$

\footnotetext{
${ }^{4}$ The general framework in which predicates are assumed to stand for concepts as unsaturated cognitive structures is called conceptualism, a formal account of which can be found in Cocchiarella 1986. A form of conceptualism in which normalized predicates are assumed to denote abstract objects is called conceptual realism, an account of which, with applications, can be found in Cocchiarella 1989.
} 
where $x_{1}, \ldots, x_{n}$ are distinct individual variables and $F^{n}$ is an $n$-place predicate variable that does not occur free in $\varphi$. (We drop the superscript on predicates when their degree is clear from the context in which they occur.) With $\lambda$ abstracts, nominalized predicates, and Leibniz's law, (LL*), applied to the latter as well, ${ }^{5}$ this principle is a consequence of the following simpler and more direct version of comprehension (subject to the same conditions):

$$
\exists F^{n}\left(F=\left[\lambda x_{1} \ldots x_{n} \varphi\right]\right) .
$$

As expressed in our present logical grammar, Frege's basic law (Vb), namely,

$$
\left[\lambda x_{1} \ldots x_{n} \varphi\right]=\left[\lambda x_{1} \ldots x_{n} \psi\right] \rightarrow \forall x_{1} \ldots \forall x_{n}(\varphi \longleftrightarrow \psi),
$$

is now an immediate consequence of $\left(\mathrm{LL}^{*}\right)$ and $(\lambda$-Conv*). It is this law that Frege himself blamed for Russell's paradox (see Frege 1964, I32), but we will have no quarrel with it here. Frege's basic law (Va) amounts to the following principle of extensionality,

$$
\forall x_{1} \ldots \forall x_{n}(\varphi \longleftrightarrow \psi) \rightarrow\left[\lambda x_{1} \ldots x_{n} \varphi\right]=\left[\lambda x_{1} \ldots x_{n} \psi\right]
$$

This principle makes explicit Frege's view that what nominalized predicates denote are the extensions of the concepts that those predicates stand for. We have no quarrel with this principle as well, but we will leave open the question of its validity and allow that nominalized predicates may denote intensional objects (the way they are normally understood in natural language). How exactly all talk of classes as the extensions of concepts is to be explained in terms of such intensional objects we will not go into here. For our purposes it will suffice to refer to the objects that nominalized predicates denote - whether those objects be extensions or intensions - simply as concept-correlates. The assumption that every ( $n$-ary) concept has a correlate (be that correlate the extension or intension of the concept), i.e., that (as abstract singular terms) nominalized predicates denote values of the bound individual variables, amounts to a form of conceptual Platonism. Formally, this assumption is stated simply as

$$
\forall F^{n} \exists x(x=F) .
$$

(Plato*)

\footnotetext{
${ }^{5} \mathrm{By}\left(\mathrm{LL}^{*}\right)$ we understand the principle $(a=b) \rightarrow(\varphi \longleftrightarrow \psi)$, where $a, b$ are singular terms, $\varphi, \psi$ are wffs (well-formed formulas), and $\psi$ comes from $\varphi$ by replacing one or more free occurrences of $b$ by free occurrences of $a$. If $a$ cannot be properly substituted for $b$ in $\varphi$ (whether because of a clash of variables or because the result is not well-formed), then we understand $\psi$ to be just $\varphi$ itself. In particular, where $n \neq k, a_{1}, \ldots, a_{n}$ are singular terms (including nominalized predicates), and $G^{k}$ is a $k$-place predicate expression, $G^{k}\left(a_{1}, \ldots, a_{n}\right)$ is not well-formed - in which case $\left(\mathrm{LL}^{*}\right)$ cannot validate $\left(F^{n}=G^{k}\right) \rightarrow\left(F^{n}\left(a_{1}, \ldots, a_{n}\right) \longleftrightarrow\right.$ $\left.G^{k}\left(a_{1}, \ldots, a_{n}\right)\right)$, because the latter is then also not well-formed. In those cases of (LL*) in which $a$ and $b$ are nominalized predicates of different degree (number of argument positions), (1) either $\psi$ is the result of replacing only free subject-position occurrences of $b$ in $\varphi$ by free subject-position occurrences of $a$, or (2) the replacement of $b$ by $a$ is not proper and (LL*) reduces to the tautologous wff $(a=b) \rightarrow(\varphi \longleftrightarrow \varphi)$. It is only when $n=k$ that, e.g.. free predicate positions of $G^{k}$ in $\varphi$ can be replaced by free predicate positions of $F^{n}$.
} 
In regard to the representation of Cantor's theorem in this context, let us adopt the following abbreviatory notation:

$$
R \text { is many-one }=_{d f} \forall x \forall y \forall z[R(x, y) \wedge R(x, z) \rightarrow y=z),
$$

and

$$
G \sqsubseteq F=_{d f} \forall x[F(x) \rightarrow G(x)] .
$$

Stated now in terms of concepts and their correlates (that may in fact be the extensions of those concepts), Cantor's theorem that any many-one relation $R$ from a concept $F$ into the concepts subordinate to $F$ (or from a class into the subclasses of that class) cannot be onto the latter can be formulated as follows:

$$
\begin{aligned}
\forall R(R \text { is many-one } & \longrightarrow \forall F(\forall x \forall G[R(x, G) \rightarrow \\
(F(x) & \longleftrightarrow G \sqsubseteq F] \rightarrow \exists G[G \sqsubseteq F \wedge \neg \exists x R(x, G)])) .
\end{aligned}
$$

Although the statement of $(\mathrm{CT})$ is relatively straightforward, we will deal in what follows with an equivalent, but somewhat simpler, thesis, which maintains only that the correlate of some concept contained in the domain of a many-one relation is not in the range of that relation:

$$
\forall R(R \text { is many-one } \rightarrow \exists F(\forall x[F(x) \rightarrow \exists y R(x, y)] \wedge \neg \exists x R(x, F))) .
$$

That is, because the validity or invalidity of $(\mathrm{CT})$ is equivalent to the validity or invalidity of $\left.\left(\mathrm{CT}_{2}\right)\right)$, we will for convenience consider only the simpler version here.

We observe first that $\left(\mathrm{CT}_{2}\right)$ has an obvious counter-instance. For, by $\left(\mathrm{CP}_{\lambda}^{*}\right)$, identity stands for a relation, i.e.,

$$
\exists R(R=[\lambda x y(x=y)]) .
$$

which means that $\left(\mathrm{CT}_{2}\right)$ can be instantiated to this relation. But then, because the identity relation is many-one, it follows from $\left(\mathrm{CT}_{2}\right)$ that some concept has a correlate that is identical with nothing, i.e., that some concept has no correlate, contrary to what is maintained by (Plato*).

On the other hand, $\left(\mathrm{CT}_{2}\right)$ is also provable on the basis of $\left(\mathrm{CP}_{\lambda}^{*}\right)$. For, where $R$ stands for any relation,

$$
\exists F(F=[\lambda x \exists G(R(x, G) \wedge \neg G(x))])
$$

is validated by $\left(\mathrm{CP}_{\lambda}^{*}\right)$. If, in addition, the relation $R$ stands for is many-one, then the correlate of the concept specified in this instance of $\left(\mathrm{CP}_{\lambda}^{*}\right)$ cannot be in the range of that relation; for, if $R(x, F)$ holds, where $F$ stands for the concept specified above, then $\neg F(x)$ must also hold — because $F(x)$ implies $\exists G[R(x, G) \wedge$ $\neg G(x)$ ], which (because the relation $R$ stands for is many-one) implies $\neg F(x)$ — and therefore $\neg \exists G[R(x, G) \wedge \neg G(x)]$ also holds, which is impossible, because $\neg F(x)$ and the reductio hypothesis $R(x, F)$ imply $\exists G[R(x, G) \wedge \neg G(x)]$. 
The upshot is that $\left(\mathrm{CT}_{2}\right)$ - and therefore $(\mathrm{CT})$ as well - is both validated and refuted in the framework in question, which means that the framework is inconsistent. Indeed, if we take the relation involved in the refutation of $\left(\mathrm{CT}_{2}\right)$, i.e., the identity relation, and put it in place of the many-one relation involved in the proof of $\left(\mathrm{CT}_{2}\right)$, the result is the concept specified in Russell's paradox, i.e., the concept of being the correlate of a concept that does not fall under that concept: $[\lambda x \exists G(R(x, G) \wedge \neg G(x))]$. Thus, by $\left(\mathrm{CP}_{\lambda}^{*}\right)$, we have the following instance of $\left(\mathrm{CP}^{*}\right)$,

$$
\exists F \forall x(F(x) \longleftrightarrow \exists G[x=G \wedge \neg G(x)]),
$$

from which, by (Plato*), it follows that the correlate of the concept in question falls under that concept if, and only if, it is the correlate of a concept under which it does not fall, which is impossible. As is clear from our earlier quote of Russell's Principles, it was in just this way, i.e., through Cantor's power-set theorem, that Russell arrived at his paradox.

\section{Frege's Double-Correlation Thesis}

The two principles that are at the heart of the above contradiction are $\left(\mathrm{CP}_{\lambda}^{*}\right)$ and (Plato*), and an appropriate restriction of either, it turns out, will suffice to avoid the contradiction. ${ }^{6}$ We need not reject the logical intuitions that are represented by the preceding Fregean-like framework, in other words, and in particular we need not reject the logical notion of a class that is implicit in those intuitions in order to overcome Russell's paradox. What we need instead is a principled way by which to restrict either $\left(\mathrm{CP}_{\lambda}^{*}\right)$ or (Plato*) without nullifying the logical intuitions of the overall framework. One such a principled way is given by Frege's double-correlation thesis.

In Frege's original framework, besides the objects that singular terms denote and the concepts that predicates stand for, there are also concepts that firstorder quantifier phrases stand for. These are called second-level concepts, as opposed to the first-level concepts that predicates stand for. Any formula with free predicate variables that can be used in the comprehension principle for such a quantifier phrase stands in effect for a second-level concept - the way, for example, $\forall x[F(x) \rightarrow G(x)]$ stands for the second-level relation of subordination between first-level concepts. Predicate quantifier phrases, and formulas that can be used to specify such a phrase, then stand for third-level concepts. ${ }^{7}$ Given variables and quantifiers for second-level concepts, we can go on in this way to describe fourth-level concepts, and then, in a similar way, fifth-level concepts, and so on through a potentially infinite hierarchy of concepts.

\footnotetext{
${ }^{6}$ The principle of $\lambda$-conversion, $\left(\lambda\right.$-Conv $\left.{ }^{*}\right)$, is also involved, but even the modified version, $\left(\exists / \lambda\right.$-Conv $\left.{ }^{*}\right)$, that we will consider later as a consequence of the modification of (Plato $\left.{ }^{*}\right)$ is applicable in the derivation of the contradiction. That is why we say that the real conflict is between $\left(\mathrm{CP}_{\lambda}^{*}\right)$ and (Plato*).

${ }^{7}$ See Frege $1964, \S 23$, for some of Frege's examples of what he means by second- and third-level concepts.
} 
What is important about this hierarchy and the way it is represented is how it differs from the hierarchy of simple types contained in Russell's ramified theory (despite the fact that Russell's initial insight into the formulation of his type theory was through Frege's hierarchy ${ }^{8}$ ). Second- and higher-level concepts, as indicated, correspond not to predicates the way that first-level concepts do, but to variable-binding operators that apply to wffs and result in wffs when so applied (such as the existential or universal quantifiers when affixed to variables). Thus, unlike predicates, operators or the type in question can be commuted and iterated and can occur within the scope of one another without regard to the levels of the concepts being represented. That is, unlike the hierarchy determined by the iterative concept of set, where the higher levels are completely determined by the lower, this hierarchy is not formed in accordance with Cantor's theorem. Rather, as indicated by the way that quantifier phrases can be commuted and occur within the scope of one another, higher-level concepts are involved in the specification of lower-level concepts no less so than lower-level concepts are involved in the specification of higher-level concepts. The interplay between these levels is so intimate, moreover, that, according to Frege, higher-level concepts can be represented in a way by lower-level concepts - so long as the lowest level of concepts can be represented by the objects that are their correlates, i.e., by their extensions (which, for us, could be their intensions instead). All higher-level concepts, in other words, can be represented by first-level concepts, which in turn, at least for Frege, can be represented by their extensions. That is why Frege - following natural language in this regard, we might add - saw no point in explicitly introducing notation for quantifying over second- and higher-level concepts.

The representation of higher-level concepts in terms of first-level concepts, and first-level concepts in terms of their extensions (or intensions), i.e., the objects that are their correlates, is most clearly seen in Frege's double-correlation thesis, according to which second-level concepts "can be represented in a certain manner" by first-level concepts whereby the concepts "that appear as arguments of the former are represented by their value-ranges," i.e., by their concept correlates (which, as already noted, may be intensions rather than extensions). ${ }^{9}$ Expressed symbolically (in the monadic case) in terms of quantifiers that range

\footnotetext{
${ }^{8}$ For a defense of this claim see Cocchiarella 1987. ch. 2. In addition to the constructive nature of Russell's ramified hierarchy, one important reason for the difference of Russell's hierarchy from Frege's was the rejection by Russell of the unsaturated nature of concepts. This led Russell to represent higher-level concepts by different types of predicate expressions (having different types of classes as their extensions), as opposed to Frege's representation in terms of different types of variable-binding operators (none of which have classes as their extensions).

${ }^{9}$ See Frege 1964, §25.
} 
over second-level (monadic) concepts, this thesis can be formulated as follows: ${ }^{10}$

$$
\forall \mathcal{Q} \exists F \forall G[\mathcal{Q} x G(x) \longleftrightarrow F(G)]
$$

Thus, for example, corresponding to the second-level concept of objectual existence, i.e., the second-level concept that first-order existential quantifier phrases stand for, there is a first-level concept of being realized such that for any firstlevel concept $G$, there exists an object that is $G$ if, and only if, the (object correlated with the) concept $G$ is realized. ${ }^{11}$

Now just as second-level concepts can be "moved down a level" (as Frege expressed it — see, e.g., 1979, 182), so too third-level concepts can be moved down a level by being correlated with second-level concepts, and fourth-level concepts can be moved down a level by being correlated with third-level concepts, and so on through the whole hierarchy of concepts. In this way Frege's entire hierarchy of concepts can be compressed and telescoped downward into the totality of first-level concepts, which in turn can be represented by their extensions (or intensions, if we drop Frege's assumption of extensionality). Thus, there are no more higher-level concepts in this framework than there are first-level concepts, just as there are no more first-level concepts than there are objects, a situation that is in direct conflict with Cantor's theorem.

Frege's double-correlation thesis represents a picture of the hierarchy of concepts that is different from the sort of hierarchy that is implicit in Cantor's theorem (and that is most clearly represented by the iterative concept of set). If such a picture is really coherent and can be given a consistent formulation, then it can be used to explain why we do not have to abandon our logical intuitions regarding classes as the extensions of concepts, i.e., our intuitions regarding the notion of a class in the logical sense, as well as why we do not have to accept Cantor's theorem. As it turns out, the picture is not only coherent but can be consistently described in two mutually supportive ways within standard second-order logic extended to include a logic of nominalized predicates as abstract singular terms.

\footnotetext{
${ }^{10}$ Frege stated this thesis for all second-level functions, not just for concept, i.e., those functions that have truth-values as their values. We ignore functions other than concepts here. It is noteworthy, incidentally, that Frege's basic law V, namely:

$$
\grave{x} F(x)=\grave{x} G(x) \rightarrow \forall x[F(x) \longleftrightarrow G(x)]
$$

is really a special case of the double-correlation thesis. For what is represented on the righthand side of this law is the second-level relation of mutual subordination between first-level concepts, which, given Frege's extensional view of concepts (as functions from objects to truthvalues), amounts to the "identity" of their concept-correlates, i.e. of their value-ranges, which is what is represented on the left-hand side.

${ }^{11}$ See "On Concept and Object", in Frege 1952, 49, where the correspondence is so tight that even the same thought is expressed by, e.g., "there is a square root of 4" and "the concept square root of 4 is realized".
} 


\section{Homogeneous Stratification and the System $\lambda$ HST $^{*}$}

Frege's double-correlation thesis, in its generalized form, amounts to a correlation first of all higher-level concepts with first-level concepts, and then to a correlation of all first-level concepts with objects, namely, their extensions or intensions, depending on whether or not we assume (Ext*), the principle of extensionality. The two mutually supportive ways of consistently formulating the picture this hierarchy presents is given by slightly modifying either of these two correlations. The first such way has to do with how first-level concepts, i.e., the concepts that predicates stand for, are specified in a standard second-order logic that has been extended to include a logic of nominalized predicates as abstract singular terms. That is, the first way has to do with a revision, or modification, of the comprehension principle, $\left(\mathrm{CP}_{\lambda}^{*}\right)$.

In starting with standard second-order logic, we assume that every instance of the comprehension principle, $\left(\mathrm{CP}_{\lambda}^{*}\right)$, that contains a $\lambda$-abstract of standard second-order logic without nominalized predicates is in itself unproblematic and should remain an instance of our revised version of $\left(\mathrm{CP}_{\lambda}^{*}\right)$. The restriction that must be imposed, accordingly, has to do only with those $\lambda$-abstracts that contain nominalized predicates, and the idea is that such $\lambda$-abstracts, in representing a stratified hierarchy of concepts that can be telescoped downward, must in principle be interpretable in terms of such a hierarchy. Minimally, this means that one must be able to assign natural numbers to all of the singular terms and predicates occurring in such a $\lambda$-abstract in such a way that the number associated with any predicate occurring as such must be the successor of the number assigned to its arguments. An additional constraint that must also be imposed is that the number assigned to any one of the arguments of a relational predicate must be the same as that assigned to any other such argument. That is, the $\lambda$-abstract must be interpretable not just in terms of a stratified hierarchy of concepts but one that is homogeneously stratified as well. This additional constraint goes beyond the requirements of Frege's original hierarchy, which given Frege's assumption that there are unequal-leveled relational concepts - was heterogeneously stratified. The constraint is necessary, however, if we are to exclude the $\lambda$-abstract $[\lambda x \exists G(R(x, G) \wedge \neg G(x))]$ that is involved in the proof of Cantor's theorem and not return in this way back to the original contradiction. For although this $\lambda$-abstract is not homogeneously stratified, it is heterogeneously stratified (and therefore is in accord with Frege's original hierarchy). ${ }^{12}$

Defined formally, we will say that a wff or $\lambda$-abstract $\varphi$ is homogeneously stratified if, and only if, there is an assignment $t$ of natural numbers to the terms and predicate expressions occurring in $\varphi$ (including $\varphi$ itself if it is a $\lambda$-abstract)

\footnotetext{
${ }^{12}$ It might also be noted in this context that predication, as an unequal-level relation between objects and the first-level concepts under which they fall, can be represented in terms or a heterogeneously stratified $\lambda$-abstract, namely, $[\lambda x y \exists G(x=G \wedge G(y))]$, from which Russell's paradox of predication can be derived. This $\lambda$-abstract is not homogeneously stratified and its exclusion by that criterion suffices to block Russell's paradox.
} 
such that (1) for all terms $a$ and $b$, if $(a=b)$ occurs in $\varphi$, them $t(a)=t(b)$; (2) for all $n \geqslant 1$, all $n$-place predicate expressions $\pi$ and all terms $a_{1}, \ldots, a_{n}$, if $\pi\left(a_{1}, \ldots, a_{n}\right)$ is a wff occurring in $\varphi$, then (i) $t\left(a_{i}\right)=t\left(a_{j}\right)$ for $1 \leq i, j \leq n$, and (ii) $t(\pi)=t\left(a_{1}\right)+1$; (3) for all natural numbers $m$, all individual variables $x_{1}, \ldots, x_{m}$, and all wffs $\psi$, if $\left[\lambda x_{1} \ldots x_{m} \psi\right]$ occurs in $\varphi$, then (i) $t\left(x_{i}\right)=t\left(x_{j}\right)$, for $1 \leq i, j \leq n$, and $t\left(\left[\lambda x_{1} \ldots x_{m} \psi\right]\right)=t\left(x_{1}\right)+1$; and (4) for all formulas $\chi$, if $[\lambda \chi]$ occurs in $\varphi$ and $a_{1}, \ldots, a_{k}$ are all of the terms or predicates occurring in $\chi$, then $t([\chi]) \geq \max \left[t\left(a_{1}\right), \ldots, t\left(a_{k}\right)\right]$.

The restriction to be imposed on $\left(\mathrm{CP}_{\lambda}^{*}\right)$, accordingly, is that it is to be restricted to $\lambda$-abstracts that are homogeneously stratified. This is to be done through a grammatical constraint on the wffs of standard second-order logic extended so as to allow for the occurrence of nominalized predicates as abstract singular terms. Because the system resulting from this constraint is a secondorder counterpart to the theory of homogeneous simple types (with $\lambda$-abstracts), we will refer to its as $\lambda \mathrm{HST}^{*}$. Thus, an expression is a meaningful wff of $\lambda \mathrm{HST}^{*}$ if, and only if, it is a wff in which every $\lambda$-abstract that occurs is homogeneously stratified. We call the restriction of $\left(\mathrm{CP}_{\lambda}^{*}\right)$ to wffs that are meaningful in $\lambda \mathrm{HST}^{*}$ the homogeneously stratified comprehension principle, or simply $\left(\mathrm{HSCP}_{\lambda}^{*}\right)$. The system $\lambda \mathrm{HST}^{*}$, accordingly, is that extension of standard second-order logic (with identity and $\lambda$-abstracts) in which (1) nominalized predicates are allowed to occur as (abstract) singular terms on a par with individual variables and other singular terms - and therefore in which (Plato*) is valid - but in which (2) the only $\lambda$-abstracts containing nominalized predicates that are allowed are those that are homogeneously stratified - and therefore in which (3) the comprehension principle $\left(\mathrm{CP}_{\lambda}^{*}\right)$ is replaced by $\left(\mathrm{HSCP}_{\lambda}^{*}\right)$.

If we were to add quantifiable variables that range over second-level concepts - i.e., variables for variable-binding operators that apply to wffs and result in wffs when so applied - then, an obvious consequence of $\left(\mathrm{HSCP}_{\lambda}^{*}\right)$ in such an extended system would be:

$$
\forall \mathcal{Q} \exists F(F=[\lambda y \exists G(y=G \wedge \mathcal{Q} x G(x))]),
$$

and therefore, by Leibniz's law, $\left(\mathrm{LL}^{*}\right)$, and $\left(\lambda\right.$-Conv $\left.{ }^{*}\right)$,

$$
\forall \mathcal{Q} \exists F \forall y(F(y) \longleftrightarrow \exists G[y=G \wedge \mathcal{Q} x G(x)]),
$$

from which, by (Plato*) and (LL*'), Frege's double-correlation thesis, (Dbl*), i.e.,

$$
\forall \mathcal{Q} \exists F \forall G[\mathcal{Q} x G(x) \longleftrightarrow F(G)]
$$

follows. The provability of the thesis in this way indicates not only the adequacy of its representation in terms of $\left(\mathrm{HSCP}_{\lambda}^{*}\right)$, but also the superfluousness of extending the system $\lambda \mathrm{HST}^{*}$ through the addition of quantifiable variables for variable-binding operators, i.e., for variables that range over second-level concepts.

Finally, let us observe that the universal concept exists as the most comprehensive concept of $\lambda \mathrm{HST}^{*}$, i.e., that by $\left(\mathrm{HSCP}_{\lambda}^{*}\right)$,

$$
\vdash_{\lambda H S T *} \exists F(F=[\lambda x(x=x)]),
$$


and therefore, by (Plato*), the universal class (or intension) exists in $\lambda \mathrm{HST}^{*}$ as a concept-correlate; that is, the universal class (or intension) exists as a legitimate totality of $\lambda \mathrm{HST}^{*}$. Yet, because identity, also by $\left(\mathrm{HSCP}_{\lambda}^{*}\right)$, stands for a relation of $\lambda \mathrm{HST}^{*}$, it follows by our earlier observation that both Cantor's theorem, $(\mathrm{CT})$, and its variant, $\left(\mathrm{CT}_{2}\right)$, are refutable in $\lambda \mathrm{HST}^{*}$, i.e.,

$$
\vdash_{\lambda H S T *} \neg(\mathrm{CT}) \wedge \neg\left(\mathrm{CT}_{2}\right) .
$$

Our earlier proof of $\left(\mathrm{CT}_{2}\right)$, and therefore indirectly of (CT) as well, cannot be given in $\lambda \mathrm{HST}^{*}$, on the other hand, because the $\lambda$-abstract needed in the proof is not available by $\left(\mathrm{HSCP}_{\lambda}^{*}\right)$. Indeed, assuming that weak Zermelo set theory is consistent, no proof of either $(\mathrm{CT})$ or $\left(\mathrm{CT}_{2}\right)$ is possible in $\lambda \mathrm{HST}^{*}$, because, as has been shown elsewhere, $\lambda \mathrm{HST}^{*}$ is consistent relative to weak Zermelo set theory (and it is equiconsistent with the latter, moreover, if (Ext*) is assumed as well). ${ }^{13}$

It should be noted, incidentally, that the exclusion of inhomogeneously stratified $\lambda$-abstracts from the meaningful wffs of $\lambda \mathrm{HST}^{*}$ does mean that all of the wffs of $\lambda \mathrm{HST}^{*}$ must be homogeneously stratified. In fact, where

$$
V={ }_{d f}[\lambda x(x=x)]
$$

we have not only the meaningfulness of $V(V)$ in $\lambda \mathrm{HST}^{*}$ but its provability as well, i.e., $\vdash_{\lambda H S T *} V(V)$.

\section{Homogeneous Stratification and the System $\mathrm{HST}_{\lambda}^{*}$}

Although Russell's paradox is formulable and therefore meaningful in $\lambda \mathrm{HST}^{*}$ as an instance of ( $\left.\mathrm{CP}^{*}\right)$, i.e., as follows,

$$
\exists F \forall x(F(x) \longleftrightarrow \exists G[x=G \wedge \neg G(x)]),
$$

it is not provable in $\lambda \mathrm{HST}^{*}$, and in fact it is refutable by Russell's argument. This is because the only instances of $\left(\mathrm{CP}^{*}\right)$ that are provable in $\lambda \mathrm{HST}^{*}$ are those that are provable on the basis of $\left(\mathrm{HSCP}_{\lambda}^{*}\right)$. In other words, we are unable to derive the above instance of $\left(\mathrm{CP}^{*}\right)$ in $\lambda \mathrm{HST}^{*}$ because the $\lambda$-abstract $[\lambda x \exists G(x=$ $G \wedge \neg G(x))]$ is not homogeneously stratified and therefore not well-formed in $\lambda \mathrm{HST}^{*}$. This means, as far as the system $\lambda \mathrm{HST}^{*}$ is concerned, that there is no concept corresponding to this $\lambda$-abstract, which is an acceptable conclusion in Frege's original framework where concepts are assumed to exist (or not to

\footnotetext{
${ }^{13}$ See Cocchiarella 1987, ch. 4, section 6. Weak Zermelo set theory is the restriction of Zermelo set theory to those instances of the Aussonderungsaxiom in which all quantifiers in the comprehension clause are restricted, i.e., of the form $(\forall x \in y) \varphi$ or $(\exists x \in y) \varphi$. Weak Z, incidentally, with or without an axiom of infinity, is equiconsistent with the theory of simple types (with or without an axiom of infinity, respectively) as a theory of classes, and so is $\lambda \mathrm{HST}^{*}+\left(\mathrm{Ext}^{*}\right)$ (with or without an axiom of infinity, respectively).
} 
exist) as unsaturated functions independently of the human capacity for concept formation.

Such a result is dubious, on the other hand, and in fact it is counter-intuitive, if by a concept we mean an unsaturated cognitive structure that is part of the overall human capacity for concept formation. After all, it is one thing to say that such a concept can have no object as its correlate (be that object an extension or an intension), but quite another to say that we cannot formulate the concept itself. It is this situation that is rectified in our second way of modifying Frege's double-correlation thesis. Thus, instead of restricting the correlation of second- and higher-level concepts with first-level concepts that is assumed in Frege's original thesis - and excluding thereby the representation of unequal higher-level concepts - our second way of consistently formulating the picture presented by the Fregean hierarchy is to modify the correlation of first-level concepts with objects. That is, instead of modifying the original comprehension principle $\left(\mathrm{CP}_{\lambda}^{*}\right)$, we can modify (Plato*) so that not every concept need be assumed to have an object as its correlate. This means that we must allow singular terms, and certain abstract singular terms in particular, to be denotationless. That is, we must revise the "standard" logic of singular terms and first-order quantifiers to one that is "free of existential presuppositions". The details of such a revision (described in section 9) are now well-known, and we shall assume it in what follows.

In "Free logic," the question as to which singular terms, if any, have a denotation, is an issue that is left completely open. Free logic allows in this regard for the possibility that there may be nothing at all in the universe of discourse that is a value or the bound individual variables. That means that we can consistently assume in the revised second-order logic in question what I have elsewhere called the Abelardian thesis that although predicates, in their role as predicates, stand for concepts as (unsaturated) cognitive structures, nominalized predicates, as abstract singular terms, denote nothing:

$$
\forall F \neg \exists x(x=F) .
$$

(Abelard*)

Whatever the merits of such a thesis, it is not relevant in a framework in which we want some, even if not all, nominalized predicates to denote conceptcorrelates. In particular, it is not relevant in a framework in which our goal is to represent the "movement downward" of Frege's hierarchy, and, in that regard, to represent at least all of the objects (if not more) that are correlates of the concepts that can be formulated in $\lambda \mathrm{HST}^{*}$. Such a representation is available, it turns out, and can be given by a first-order counterpart of the homogeneously stratified comprehension principle, $\left(\mathrm{HSCP}_{\lambda}^{*}\right)$, which is described as follows:

$$
\exists y\left(a_{1}=y\right) \wedge \ldots \wedge \exists y\left(a_{k}=y\right) \rightarrow \exists y\left(y=\left[\lambda x_{1} \ldots x_{n} \varphi\right]\right) . \quad\left(\exists / \mathrm{HSCP}_{\lambda}^{*}\right)
$$

where (1) $y$ is an individual variable not occurring free in $\varphi ;(2)\left[\lambda x_{1} \ldots x_{n} \varphi\right]$ is homogeneously stratified; (3) $a_{1}, \ldots, a_{n}$ are all of the variables or nonlogical constants occurring free in $\left[\lambda x_{1} \ldots x_{n} \varphi\right]$; and (4) all bound predicate variables in $\varphi$ are bound to objects (i.e., for all predicate variables $F$ and wffs $\psi$, if $\forall F \psi$ 
occurs in $\varphi$, then $\psi$ is of the form $\exists z(F=z) \rightarrow \chi$, for some wff $\chi)$. Because of its connection with the system $\lambda \mathrm{HST}^{*}$, we refer to the new system in question as $\operatorname{HST}_{\lambda}^{*}$.

In $\mathrm{HST}_{\lambda}^{*}$, it should be noted, an inhomogeneously stratified $\lambda$-abstract of the form, $[\lambda x \exists G(R(x, G) \wedge \neg G(x))]$ — and in particular the $\lambda$-abstract $[\lambda x \exists G(x=$ $G) \wedge \neg G(x))$ ] of Russell's paradox — is not only grammatically well-formed, but, by $\left(\mathrm{CP}_{\lambda}^{*}\right)$, is posited as standing, in its role as a predicate, for an unsaturated concept as well. What Russell's argument shows is not that this predicate fails to stand for a concept but rather that when nominalized it fails to denote an object. That is, instead of a contradiction, we have both

$$
\left.\vdash_{H S T_{\lambda}^{*}} \exists F(F=[\lambda x \exists G(x=G) \wedge \neg G(x))]\right),
$$

and

$$
\left.\vdash_{H S T_{\lambda}^{*}} \neg \exists y(y=[\lambda x \exists G(x=G) \wedge \neg G(x))]\right) .
$$

This means, in particular, that (Plato*) is refutable in $\mathrm{HST}_{\lambda}^{*}$, i.e.,

$$
\exists F \forall x(x \neq F) .
$$

The refutation of (Plato*) indicates that Frege's double-correlation thesis is provable in $\mathrm{HST}_{\lambda}^{*}$ (when the latter is supplemented with bindable variables for second-level concepts), but only in a restricted form. In particular, given the following abbreviatory notation for restricted predicate quantifiers (where $G$ is any monadic predicate variable and $\varphi$ any wff),

$$
\begin{gathered}
(\forall G / O b j) \varphi={ }_{d f} \forall G(\exists x(x=G) \rightarrow \varphi), \\
(\exists G / O b j) \varphi=_{d f} \exists G(\exists x(x=G) \wedge \varphi),
\end{gathered}
$$

then the restricted form of Frege's double-correlation thesis that is provable in $\mathrm{HST}_{\lambda}^{*}$ can be stated as follows:

$$
\forall \mathcal{Q} \exists F(\forall G / O b j)(\mathcal{Q} x G(x) \longleftrightarrow F(G)) .
$$

The relationship between the two systems $\lambda \mathrm{HST}^{*}$ and $\mathrm{HST}_{\lambda}^{*}$ can best be seen in the following Iemma. ${ }^{14}$

Lemma: If $\varphi$ is a meaningful wff of $\lambda \mathrm{HST}^{*}$ in which all predicate quantifiers are restricted as above, $y$ is an individual variable not occurring in $\varphi$, and $a_{1}, \ldots, a_{k}$ are all of the variables or nonlogical constants occurring free in $\varphi$, then $\vdash_{H S T_{\lambda}^{*}} \varphi$ only if $\vdash_{H S T_{\lambda}^{*}} \exists y\left(a_{1}=y\right) \wedge \ldots \wedge \exists y\left(a_{k}=y\right) \rightarrow \varphi$.

It follows from this lemma that any sentence of $\lambda \mathrm{HST}^{*}$ in which no nonlogical constants occur and that is bound to objects (i.e., in which all predicate quantifiers are restricted as above) is a theorem of $\lambda \mathrm{HST}^{*}$ only if it is also a theorem of $\mathrm{HST}_{\lambda}^{*}$. But, by (Plato*), every meaningful sentence of $\lambda \mathrm{HST}^{*}$ is

\footnotetext{
${ }^{14}$ See Cocchiarella 1986 , ch. $5, \S 4$, for proof of this lemma.
} 
provably equivalent in $\lambda \mathrm{HST}^{*}$ to a sentence that is bound to objects, and therefore, in the sense of this equivalence, $\mathrm{HST}_{\lambda}^{*}$ may be said to contain $\lambda \mathrm{HST}^{*}$. In particular, by $\left(\exists / \mathrm{HSCP}_{\lambda}^{*}\right)$, every object that is a correlate of a concept posited in $\lambda \mathrm{HST}^{*}$ is also a correlate of a concept posited in $\mathrm{HST}_{\lambda}^{*}$. Thus, because the universal concept, $V$, has a correlate (be it an extension or an intension) in $\lambda \mathrm{HST}^{*}$, then it also has a correlate in $\mathrm{HST}_{\lambda}^{*}$; and therefore such a correlate itself falls under the universal concept, i.e.,

$$
\vdash_{H S T_{\lambda}^{*}} V(V) .
$$

Finally, it should also be noted that $\mathrm{HST}_{\lambda}^{*}$ can also be proved consistent relative to weak Zermelo set theory (or the theory of simple types as a theory of classes, and it is equiconsistent with both if (Ext*) is assumed as well). ${ }^{15}$

In regard to the status of Cantor's theorem (CT), or its equivalent but more simply stated alternative, $\left(\mathrm{CT}_{2}\right)$, let us first note that the refutation given earlier Section 2, which depended upon (Plato*), no longer applies. Indeed, if $R$ is the identity relation, $[\lambda x v(x=y)]$, as in that earlier refutation, then there is a concept $F$ - e.g., the Russellian concept, $[\lambda x \exists G(R(x, G) \wedge \neg G(x))]$ — such that $\forall x(F(x) \rightarrow \exists y R(x, y)]$ and $\neg \exists x R(x, F)$, i.e., $\neg \exists x(x=F)$, are both provable in $\mathrm{HST}_{\lambda}^{*}$. Thus, not only are $(\mathrm{CT})$ and $\left(\mathrm{CT}_{2}\right)$ not refutable in $\mathrm{HST}_{\lambda}^{*}$, but in some of their instances both are provable in $\mathrm{HST}_{\lambda}^{*} \cdot{ }^{16}$

The fact that (Plato*) is refutable in $\mathrm{HST}_{\lambda}^{*}$ indicates that another version of Cantor's theorem, namely,

$$
\begin{aligned}
& \forall R(\forall F \forall G \forall x[R(x, F) \wedge R(x, G) \quad \rightarrow \\
&\forall y(F(y) \quad \longleftrightarrow \quad G(y))] \rightarrow \exists F \neg \exists x R(x, F)),
\end{aligned}
$$

which says that no relation that correlates coextensive concepts with the same object (or, what amounts to the sane idea, that correlates the extensions of concepts with objects) correlates (the extension of) each concept with some object, is vacuously provable in $\mathrm{HST}_{\lambda}^{*} \cdot{ }^{17}$ This is because in the free logic of $\mathrm{HST}_{\lambda}^{*}$ only "real" objects, i.e., objects that are values of the bound individual variables, can fall under a concept, including a relational concept. That is, where $y$ is not free in either $a$ or $b,{ }^{18}$

$$
\vdash_{H S T_{\lambda}^{*}} \forall R[R(a, b) \rightarrow \exists y(a=y) \wedge \exists y(b=y)],
$$

\footnotetext{
${ }^{15}$ See Cocchiarella 1987, ch. $4, \S 7$. The equiconsistency depends upon an axiom of infinity well if such an axiom is assumed to be part of Weak $\mathrm{Z}$ or of the theory of simple types.

${ }^{16}$ For the proof of an instance of $(\mathrm{CT})$ in $\mathrm{HST}_{\lambda}^{*}$, we need only let $R$ be the identity relation and $F$ be the universal concept, $[\lambda x(x=x)]$. We then observe that $\forall x \forall G(R(x, G) \rightarrow$ $[F(x) \longleftrightarrow G \sqsubseteq F])$ is provable and that there is a concept $G-$ e.g., $[\lambda x \exists G(R x, G) \wedge \neg G(x))]$ - such that $G \sqsubseteq F$ and $\neg \exists x R(x, G)$, i.e., $\neg \exists x(x=G)$, are both provable in HST ${ }_{\lambda}^{*}$.

${ }^{17}$ This version of Cantor's theorem, or rather a statement essentially equivalent to $\left(\mathrm{CT}_{3}\right)$, was first suggested to me by Stewart Shapiro.

${ }^{18}$ This result follows from $[\lambda x y R(x, y)]=R$, which is an instance of $\left(\operatorname{id}_{\lambda}^{*}\right)$, and the free logic version of $\left(\lambda\right.$-Conv $\left.{ }^{*}\right)$, namely,

$$
\left.\left[\lambda x_{1} \ldots x_{n} \varphi\right]\left(a_{1}, \ldots, a_{n}\right) \longleftrightarrow \exists x_{1} \ldots \exists x_{n}\left(a_{1}=x_{1}\right) \wedge \ldots \wedge \exists x_{n}\left(a_{n}=x_{n}\right) \wedge \varphi\right),
$$

where no $x_{i}$ is free in any $a_{j}$, for all $i, j$ such that $1 \leq i, j \leq n$.
} 
and therefore, given the refutation of (Plato*),

$$
\left.\vdash_{H S T_{\lambda}^{*}} \forall R \exists F \neg \exists x R x, F\right)
$$

from which follows the vacuous provability of $\left(\mathrm{CT}_{3}\right)$ in $\mathrm{HST}_{\lambda}^{*}$. Of course, in $\lambda$ HST, where (Plato*) is valid, $\left(\mathrm{CT}_{3}\right)$ is not provable but refutable by taking $R$ to be the identity relation.

There are stronger forms of Cantor's theorem, $(\mathrm{CT})$, and it variants, $\left(\mathrm{CT}_{2}\right)$ and $\left(\mathrm{CT}_{3}\right)$, that are refutable in $\mathrm{HST}_{\lambda}^{*}$ however. These versions depend upon the existential predicate quantifiers in the consequents of $(\mathrm{CT}),\left(\mathrm{CT}_{2}\right)$, and $\left(\mathrm{CT}_{3}\right)$ being restricted in the manner indicated above. Thus, where

$$
\begin{aligned}
\forall R(R \text { is many-one } \rightarrow \quad \forall F[\forall F \forall G(R(x, G) \rightarrow(F(x) \longleftrightarrow \\
G \sqsubseteq F)] \rightarrow \neg(\exists G / O b j)[G \sqsubseteq F \wedge \neg \exists x R(x, G)]), \\
\forall R(R \text { is many-one } \rightarrow \quad(\exists F / O b j)[\forall x(F(x) \rightarrow \\
\exists y R x, y)) \wedge \neg \exists R(x, F)]), \\
\forall R(\forall F \forall G \forall x[R(x, F) \wedge R(x, G) \rightarrow \forall y(F(y) \longleftrightarrow G(y))] \\
\rightarrow \quad(\exists F / O b j) \neg \exists x R(x, F)),
\end{aligned}
$$

then it is easily seen that each of these forms of Cantor's theorem is refutable in $\operatorname{HST}_{\lambda}^{*}$. That is,

$$
\vdash_{H S T_{\lambda}^{*}} \neg(\exists / \mathrm{CT}) \wedge \neg\left(\exists / \mathrm{CT}_{2}\right) \wedge \neg\left(\exists / \mathrm{CT}_{3}\right) .
$$

(In the refutation of each of these forms of Cantor's theorem we need only take $R$ to be the identity relation, i.e., $[\lambda x y(x=y)]$, and observe that by definition $(\forall F / O b j) \exists x R(x, F)$, i.e., $(\forall F / O b j) \exists x(F=x)$, is trivially provable in $\mathrm{HST}_{\lambda}^{*} \cdot{ }^{19}$

Each of these refutable forms of Cantor's theorem is stronger than its counterpart in $\mathrm{HST}_{\lambda}^{*}$, because each implies but is not equivalent to its counterpart. Of course, in $\lambda \mathrm{HST}^{*}$ where (Plato*) is provable, each form is equivalent to its counterpart - but then, each of the counterparts is refutable in $\mathrm{HST}_{\lambda}^{* *}$. The counterparts are not refutable in $\mathrm{HST}_{\lambda}^{*}$, to be sure, but that is only because they have instances that are vacuously provable, which can hardly lend any substantive support to Cantor's theorem in such a framework. The proper content of

\footnotetext{
${ }^{19}$ Given that $R$ is the identity relation, then for the refutation of $\left(\exists / \mathrm{CT}_{2}\right)$ and $\left(\exists / \mathrm{CT}_{3}\right)$ in $\operatorname{HST}_{\lambda}^{*}$, we have it that not only is $(\forall F / O b j) \exists x R(x, F)$ provable but so are $\forall F \forall x[F(x) \rightarrow$ $\exists y R(x, y)]$ and, by Leibniz's law, (LL*),

$$
\forall F \forall G \forall x(R(x, F) \wedge R(x, G) \rightarrow \forall y[F(y) \longleftrightarrow G(y)]),
$$

For the refutation or $(\exists / \mathrm{CT})$, let $F$ be the universal concept, $[\lambda x(x=x)]$, and observe that because $(\forall G / O b j) \exists x R(x, G)$ is provable, where $R$ is the identity relation, then so is $(\forall G / O b j)[G \sqsubseteq F \rightarrow \exists x R(x, G)]$.
} 
Cantor's theorem and its variants, in other words, is really that given by the stronger forms (which, as noted, are not really stronger in $\mathrm{HST}_{\lambda}^{*}$ ). In any case, it is in the sense of these stronger forms that we maintain that Cantor's theorem is refutable in a logical framework that is based upon Frege's double-correlation thesis. The consistency of these frameworks shows that its not our logical intuitions (including in particular the logical notion of a class as the extension of a concept) that must be given up, but, assuming that we adopt either $\lambda \mathrm{HST}^{*}$ or HST $_{\lambda}^{*}$ as our logical framework, Cantor's power-set theorem instead.

\section{Identity and the system $\lambda \mathrm{T}^{*}$}

The conflict between Cantor's theorem and our logical intuitions, especially those regarding the existence of the universal class (or intension), is not absolute, it turns out, but can be resolved by turning to a different view of identity; namely, one according to which identity, as a logical, syncategorematic constant, stands for a "transcendental" (or cross-categorial) notion that applies to concepts as well as to objects, and therefore a notion that is not itself a relation (between objects). The assumption that identity stands for a relation, i.e., that as an instance of the comprehension principle $\left(\mathrm{CP}_{\lambda}^{*}\right)$, we have

$$
\exists R(R=[\lambda y(y=y)]),
$$

was an essential step in the refutation of Cantor's theorem given in section 2. If we drop this assumption by excluding as well-formed all $\lambda$-abstracts in which the identity sign occurs, then it turns out that the refutation of Cantor's theorem fails, and therefore that Russell's paradox fails as well. The system that results from standard second-order logic with $\lambda$-abstracts and nominalized predicates as abstract singular terms by excluding those $\lambda$-abstracts in which the identity sign occurs we call $\lambda \mathrm{T}^{*}$. We refer to the restricted version of $\left(\left(\mathrm{CP}_{\lambda}^{*}\right)\right.$ that holds in $\lambda \mathrm{T}^{*}$ as $\left(\mathrm{CP}_{\lambda}^{* *}\right)$.

Russell's paradox, as an instance of $\left(\mathrm{CP}^{*}\right)$, i.e., the Russellian concept specified as follows,

$$
\exists F \forall x(F(x) \longleftrightarrow \exists G[x=G \wedge \neg G(x)]),
$$

is not derivable in $\lambda \mathrm{T}^{*}$, though it is well-formed. The only instances of $\left(\mathrm{CP}^{*}\right)$ that are derivable in $\lambda \mathrm{T}^{*}$ are those that can be derived from $\left(\mathrm{CP}_{\lambda}^{* *}\right)$, i.e., those in which the identity sign does not occur. Of course, instead of identity we can turn to indiscernibility, which, for convenience, we will abbreviate as follows (where $a, b$ are arbitrary terms, simple or complex, and $F$ is a monadic predicate variable new to $a$ and $b$ ),

$$
a \equiv b=_{d f} \forall F[F(a) \longleftrightarrow F(b)] .
$$

In other words, even if identity is not a relation, indiscernibility is, and therefore, by $\left(\mathrm{CP}_{\lambda}^{* *}\right)$, we have

$$
\vdash_{\lambda T^{*}} \exists R(R=[\lambda x \exists G(x \equiv G \wedge \neg G(x))]),
$$


from which, by (LL*) and elementary transformations, we have the modified Russell concept specified as follows:

$$
\vdash_{\lambda T^{*}} \exists F \forall x(F(x) \longleftrightarrow \exists G[x \equiv G \wedge \neg G(x)]) .
$$

This last result does not lead to Russell's paradox, however. That is, no contradiction based on this wff is forthcoming - nor can a contradiction be constructed in $\lambda \mathrm{T}^{*}$ in any other way. The system $\lambda \mathrm{T}^{*}$ is consistent, in other words, and it can be proved consistent relative to standard second-order logic with identity and $\lambda$-abstracts. ${ }^{20}$

The attempt to derive Russell's paradox from the above result does show by (Plato*) that the modified Russellian concept in question applies to itself, or, strictly speaking, that the correlate of any concept coextensive with the modified Russell concept falls under that concept:

$$
\vdash_{\lambda T^{*}} \forall x(F(x) \longleftrightarrow \exists G[x \equiv G \wedge \neg G(x)]) \rightarrow F(F) .
$$

It does not show, however, that the correlate of any such concept also does not fall under that concept, which would be the contradiction one might expect. Rather, what follows instead is that the correlate of such a concept is indiscernible from the correlate of a concept under which it does not fall, and therefore that

$$
\vdash_{\lambda T^{*}} \exists F \exists G[F \equiv G \wedge F(F) \wedge \neg G(F)] .
$$

From this it follows by (Plato*) that there are non-coextensive concepts in $\lambda \mathrm{T}^{*}$ whose correlates are indiscernible:

$$
\vdash_{\lambda T^{*}} \exists F \exists G(F \equiv G \wedge \neg \forall y[F(y) \longleftrightarrow G(y)]) .
$$

This last result is not as odd as it seems, because what it really amounts to is an instance of Cantor's theorem in the form $\left(\mathrm{CT}_{3}\right)$. To see this, let us first note that by instantiating $\left(\mathrm{CT}_{3}\right)$ to the indiscernibility relation, we have

$$
\forall F \forall G \forall x(x \equiv F \wedge x \equiv G \rightarrow \forall y[F(y) \longleftrightarrow G(y)] \rightarrow \exists F \neg \exists x(x \equiv F) .
$$

But, by (Plato*) and the fact that identity implies indiscernibility,

$$
\vdash_{\lambda T^{*}} \neg \forall F \neg(x \equiv F),
$$

and therefore, assuming that $\left(\mathrm{CT}_{3}\right)$ is valid $\lambda \mathrm{T}^{*}$, we have

$$
\vdash_{\lambda T^{*}} \exists F \exists G \exists x(x \equiv F \wedge x \equiv G \wedge \neg \forall y[F(y) \longleftrightarrow G(y)]),
$$

which, because indiscernibility is an equivalence relation (and in fact the strongest equivalence relation in $\lambda \mathrm{T}^{*}$ ), reduces to the above thesis that there are noncoextensive concepts in $\lambda \mathrm{T}^{*}$ whose correlates are indiscernible.

\footnotetext{
${ }^{20}$ See Cocchiarella $1986, \mathrm{ch} .4$, section 6 . The proof of consistency given is for $\mathrm{T}^{*}$, which is $\lambda \mathrm{T}^{*}$ without $\lambda$-abstracts, but it can easily be extended to deal with $\lambda$-abstracts as well.
} 
The above result is not odd, we maintain, not only because it is an instance of $\left(\mathrm{CT}_{3}\right)$, but because $\left(\mathrm{CT}_{3}\right)$ itself - and Cantor's theorem, $(\mathrm{CT})$, and it variant, $\left(\mathrm{CT}_{2}\right)$ as well - is provable in $\lambda \mathrm{T}^{*}$. Thus, by way of an informal sketch of a proof of $\left(\mathrm{CT}_{3}\right)$, let $R$ be any relation for which the antecedent of $\left(\mathrm{CT}_{3}\right)$ is true and its consequent false, i.e., for which $\forall F \exists x R(x, F)$ is true as well. Then, where $F$ is the concept that $[\lambda x \exists G(R(x, G) \wedge \neg G(x))]$ stands for - which, by $\left(\mathrm{CP}_{\lambda}^{* *}\right)$, we know exists in $\lambda \mathrm{T}^{*}$ - it follows by the reductio assumption that $R(x, F)$ is true of some object as a value of $x$. But, for any such object, $F(x)$ must be true; for if $F(x)$ were false, then, by ( $\lambda$-Conv $\left.{ }^{*}\right), \forall G[R(x . G) \rightarrow G(x)]$ would be true, and therefore, by universally instantiating $G$ to $F, F(x)$ would be true after all. But then, because $F(x)$ is true, it follows by $\left(\lambda\right.$-Conv $\left.{ }^{*}\right)$ that $[R(x, G) \wedge \neg G(x)]$ must also be true of some concept $G$, and therefore, by assumption of the antecedent of $\left(\mathrm{CT}_{3}\right), \forall y[F(y) \longleftrightarrow G(y)]$ must also true. This last is impossible, however, because $F(x)$ is true, and therefore $G(x)$ is true as well, whereas we already have it that $\neg G(x)$ is true. Thus, the assumption that there is a relation for which the antecedent of $\left(\mathrm{CT}_{3}\right)$ is true and its consequent false leads in $\lambda \mathrm{T}^{*}$ to an impossible situation, which shows that $\left(\mathrm{CT}_{3}\right)$ is provable in $\lambda \mathrm{T}^{*}$.

Our earlier proof in section 2 of $\left(\mathrm{CT}_{2}\right)$ is easily seen to apply in $\lambda \mathrm{T}^{*}$, and, as noted there, a similar proof applies to Cantor's theorem, (CT), as well. In addition, because (Plato*) is provable in $\lambda \mathrm{T}^{*}$, it follows that all of the stronger forms, $(\exists / \mathrm{CT}),\left(\exists / \mathrm{CT}_{2}\right)$, and $\left(\exists / \mathrm{CT}_{3}\right)$ of Cantor's theorem are also provable in $\lambda \mathrm{T}^{*}$ (because they are not really stronger than their counterparts in $\lambda \mathrm{T}^{*}$ after all but rather are equivalent to them). Thus, in $\lambda \mathrm{T}^{*}-$ unlike the situation in either $\lambda \mathrm{HST}^{*}$ or $\mathrm{HST}_{\lambda}^{*}$ - all forms of Cantor's theorem are provable, i.e.,

$$
\vdash_{\lambda T^{*}}(\mathrm{CT}) \wedge\left(\mathrm{CT}_{2}\right) \wedge\left(\mathrm{CT}_{3}\right) .
$$

Now Frege's double-correlation thesis, $\left(\mathrm{Dbl}^{*}\right)$, we have argued, means that Cantor's theorem must be rejected. It is natural to expect, accordingly, that if Cantor's theorem is not rejected, but in fact provable in all of its forms, then Frege's double-correlation thesis must be rejected instead. Frege's thesis must be refutable in $\lambda \mathrm{T}^{*}$, in other words, when $\lambda \mathrm{T}^{*}$ is supplemented with variables and a quantifier logic for second-level concepts. For, if Frege's thesis were to hold, then concepts whose correlates (be they extensions or intensions) are indiscernible with respect to first-level concepts would themselves be indiscernible with respect to second-level concepts, i.e., then

$$
\forall \mathcal{Q} \exists F \forall G[\mathcal{Q} x G(x) \longleftrightarrow F(G)] \vdash_{\lambda T^{*}} \forall F \forall G(F \equiv G \rightarrow \forall \mathcal{Q}[(\mathcal{Q} x F(x) \longleftrightarrow
$$
$\mathcal{Q} x G(x)])$

would be true.

But in a quantifier logic for second- as well as first-level concepts, firstlevel concepts that are indiscernible with respect to second-level concepts are coextensive, i.e.,

$$
\forall \mathcal{Q}[\mathcal{Q} x F(x) \longleftrightarrow G(x)] \rightarrow \forall y[F(v) \longleftrightarrow G(y)]
$$

is provable in such a logic. ${ }^{21}$ Therefore, Frege's double-correlation thesis has the

\footnotetext{
${ }^{21}$ Briefly, the argument for this is that by universal instantiation and tautologous transfor-
} 
consequence that concepts whose correlates are indiscernible are coextensive, i.e.,

$$
\forall \exists F \forall G[\mathcal{Q} x G(x) \longleftrightarrow F(G)] \vdash_{\lambda T^{*}} \forall F \forall G(F \equiv G \rightarrow \forall y[F(y) \longleftrightarrow G(y)] .
$$

But, as noted above it is just this consequence that is refuted by $\left(\mathrm{CT}_{3}\right)$; and $\left(\mathrm{CT}_{3}\right)$, as already noted, is provable in $\lambda \mathrm{T}^{*}$. Thus, when $\lambda \mathrm{T}^{*}$ is supplemented with a logic for second-level concepts, we have a refutation of (Dbl*), i.e.,

$$
\vdash_{\lambda T^{*}} \neg \forall \mathcal{Q} \exists F \forall G[\mathcal{Q} x G(x) \longleftrightarrow F(G)] .
$$

This last result is appropriate for $\lambda \mathrm{T}^{*}$, it should be emphasized, because the refutation in this system of the thesis that concepts whose correlates are indiscernible are themselves coextensive amounts to a rejection of the logical picture implicit in Frege's hierarchy - namely, that higher-level concepts are as much involved in the determination of lower-level concepts as the latter are in the determination of the former, which means that concepts whose correlates are indiscernible should themselves be (at least) coextensive. Thus, in a system where the latter thesis is rejected on the basis of Cantor's theorem, it is natural to expect that Frege's thesis will also be rejected.

Note that because of the occurrence of the identity sign in the $\lambda$-abstract $[\lambda y \exists G(y=G \wedge \mathcal{Q} x G(x))]$, the proof we gave for Frege's double-correlation thesis in $\lambda$ HST $^{*}$ does not apply in $\lambda \mathrm{T}^{*}$. The replacement of the identity sign in this $\lambda$-abstract by the sign for the indiscernibility relation does not result in Frege's thesis, moreover, but in something much weaker (and consistent) — just as replacing identity by indiscernibility in the formulation for Russell's paradox results in something much weaker (and consistent), namely, an application of Cantor's theorem in the form $\left(\mathrm{CT}_{3}\right)$. This shows how different identity is from indiscernibility, because what it says is that not all concepts whose correlates are indiscernible are coextensive, whereas identity, as a "transcendental" (or cross-categorial) notion regarding concepts as well as objects, requires identical concepts to be coextensive. Thus, what follows in $\lambda \mathrm{T}^{*}$ is that different concepts can have indiscernible objects as their correlates, i.e.,

$$
\vdash_{\lambda T^{*}} \exists F \exists G[F \neq G \wedge F \equiv G] .
$$

From this it follows that in $\lambda \mathrm{T}^{*}$ indiscernibility is not a many-one relation and that identity does not, indeed cannot, stand for a relation, i.e.,

$$
\begin{gathered}
\vdash_{\lambda T^{*}} \neg \exists R \forall x \forall y[R(x, y) \longleftrightarrow x=y] . \\
\hline \text { mations, } \\
\quad \forall G[\mathcal{Q} x G(x) \longleftrightarrow G(y)] \rightarrow([\mathcal{Q} x F(x) \longleftrightarrow \mathcal{Q} x G(x)] \rightarrow[F(y) \longleftrightarrow G(y)])
\end{gathered}
$$

is valid, and therefore, by universal generalization and elementary quantifier laws, so is

$$
\forall y \exists \mathcal{Q} \forall G[\mathcal{Q} x G(x) \longleftrightarrow G(y)] \rightarrow(\forall \mathcal{Q}[\mathcal{Q} x F(x) \longleftrightarrow \mathcal{Q} x G(x)] \rightarrow \forall y[F(y) \longleftrightarrow G(y)]) .
$$

But, by the comprehension principle for second-level concepts,

$$
\forall y \exists \mathcal{Q} \forall G[\mathcal{Q} x G(x) \longleftrightarrow G(y)]
$$

is valid, and therefore so is the consequent that first-level concepts are coextensive if they are indiscernible with respect to second-level concepts. 
Because the identity sign does not stand for a relation in $\lambda \mathrm{T}^{*}$, the universal class (or intension), $V$, as defined earlier, namely, as $[\lambda x(x=x)]$ is no longer available. Instead we must turn to a weaker version defined in terms of indiscernibility:

$$
\mathcal{U}={ }_{d f}[\lambda x(x \equiv x)] .
$$

This version is distinctively weaker in $\lambda \mathrm{T}^{*}$ than the original in that, together with Cantor's theorem, it does not yield Cantor's paradox. Indiscernibility, in falling short of identity, does not itself amount to a many-one relation, and in that regard it cannot be used to correlate each object in (or falling under) $\mathcal{U}$ with a "subclass" of $\mathcal{U}$ (e.g., its "singleton") in order to show that there are cardinally fewer objects falling under It than there are "subclasses" of $\mathcal{U}$. Identity, were it allowed to occur in $\lambda$-abstracts, could be used to define such a correlation - namely as the many-one relation that $[\lambda x y(y=[\lambda z(z \equiv x)])]$ would then stand for - but such a specification is not possible in $\lambda \mathrm{T}^{*}$. Thus, $\lambda \mathrm{T}^{*}$ provides an example of a system in which, though Cantor's theorem is provable, Cantor's paradox is not.

\section{$7 \quad$ Identity and the system $\mathbf{T}_{\lambda}^{*}$}

One way in which identity can be allowed to occur in $\lambda$-abstracts and thereby represent a (many-one) relation is to modify $\lambda \mathrm{T}^{*}$ the way we modified $\lambda \mathrm{HST}^{*}$ to obtain $\mathrm{HST}_{\lambda}^{*}$ - namely, by allowing all $\lambda$-abstracts to be well-formed, including those in which the identity sign occurs, but then avoid Russell's paradox by dropping the requirement that all nominalized predicates must denote as abstract singular terms. Thus, as in the case of the system HST ${ }_{\lambda}^{*}$, instead of modifying the original comprehension principle $\left(\mathrm{CP}_{\lambda}^{*}\right)$, we can instead modify the thesis (Plato*) that every concept must have a concept-correlate (be it an extension or an intension).

Instead of (Plato*) we follow the strategy adopted in the development of the system $\mathrm{HST}_{\lambda}^{*}$ and assume a first-order counterpart of the restricted comprehension principle $\left(\mathrm{CP}_{\lambda}^{*}\right)$. That is, in analogy with $\left(\exists / \mathrm{HSCP}_{\lambda}^{*}\right)$, the first-order counterpart of the comprehension principle $\left(\mathrm{CP}_{\lambda}^{*}\right)$, we assume the following as a first-order counterpart to $\left(\mathrm{CP}_{\lambda}^{*}\right)$ :

$$
\exists y\left(a_{1}=y\right) \wedge \ldots \wedge \exists y\left(a_{k}=y\right) \rightarrow \exists y\left(y=\left[\lambda x_{1} \ldots x_{n} \varphi\right]\right), \quad\left(\exists / \mathrm{CP}_{\lambda}^{* *}\right)
$$

where (1) $y$ is an individual variable not occurring free in $\varphi,(2) \varphi$ is an identityfree wff, (3) $a_{1}, \ldots, a_{k}$ are all of the variables or nonlogical constants occurring free $\left[\lambda x_{1} \ldots x_{n} \varphi\right]$, and (4) all bound predicate variables in $\varphi$ are bound to objects (i.e., $\forall F \psi$ occurs in $\varphi$, then $\psi$ is of the form $\exists z(F=z) \rightarrow \chi$, for some wff $\chi$. Again, because of its connection with the system $\lambda \mathrm{T}^{*}$, we refer to the new system in question as $\mathrm{T}_{\lambda}^{*}$.

By the unqualified comprehension principle $\left(\mathrm{CP}_{\lambda}^{*}\right)$, it is provable in $\mathrm{T}_{\lambda}^{*}$ that there is such a relational concept as identity, i.e.,

$$
\vdash_{T_{\lambda}^{*}} \exists R(R=[\lambda x y(x=y)]),
$$


but it is not provable in $\mathrm{T}_{\lambda}^{*}$ that this concept has an extension (or intension), i.e., that it has a concept-correlate. In fact, it is provable in $\mathrm{T}_{\lambda}^{*}$ that it cannot have any correlate at all, i.e.,

$$
\vdash_{T_{\lambda}^{*}} \neg \exists z(z=[\lambda x y(x=y)]) .
$$

For if there were such a correlate, then, by $\left(\exists / \mathrm{CP}_{\lambda}^{* *}\right)$,

$$
\exists y(y=[\lambda x(\exists G / O b j)(x=G \wedge \neg G(x))])
$$

would be true, from which Russell's paradox would follow, and $\mathrm{T}_{\lambda}^{*}$ unlike $\lambda \mathrm{T}^{*}$, would then be inconsistent. But just as $\mathrm{HST}_{\lambda}^{*}$ can be shown to be consistent relative to $\lambda \mathrm{HST}^{*}, \mathrm{~T}_{\lambda}^{*}$ can be shown to be consistent relative to $\lambda \mathrm{T}^{*}$, which means that no contradiction is forthcoming in $\mathrm{T}_{\lambda}^{*}$ at all.

Cantor's theorem (CT) and its variant $\left(\mathrm{CT}_{2}\right)$ are provable in by essentially the same argument that shows their provability in $\lambda \mathrm{T}^{*} .\left(\mathrm{CT}_{3}\right)$ is provable as well, but only vacuously, because the consequent of ( $\left.\mathrm{CT}_{3}\right)$, namely, $\exists F \neg R(x, F)$, is already provable in $\mathrm{T}_{\lambda}^{*}$ for those concepts (such as the Russellian concept described above) that have no object as a concept-correlate.

The stronger version of $\left(\mathrm{CT}_{3}\right)$, namely, $\left(\exists / \mathrm{CT}_{3}\right)$, is not provable in $\mathrm{T}_{\lambda}^{*}$ unless the many-one relation in question is also assumed to have a concept-correlate - and the same observation applies to the stronger versions of $(\mathrm{CT})$ and $\left(\mathrm{CT}_{2}\right)$ as well. But, as already noted, the identity relation - which is the relation that is needed for the derivation of Cantor's paradox - does not have a conceptcorrelate (be it an extension or an intension) in $\mathrm{T}_{\lambda}^{*}$, and for that reason Cantor's paradox is not derivable in $\mathrm{T}_{\lambda}^{*}$. Thus even when $\lambda \mathrm{T}^{*}$ is modified to allow for identity as a relational concept, i.e., when $\lambda \mathrm{T}^{*}$ is replaced by $\mathrm{T}_{\lambda}^{*}$, we are still unable to derive Cantor's paradox. For even though every concept is related to itself by the relation of identity - where identity is a transcendental notion applying in different categories - not every concept has an extension (or intension), and therefore not every concept has a concept-correlate that is also related to itself by the relation of identity. In this way the proof of Cantor's theorem, or of any of it variants, as it applies to concepts does not carry over to objects as well, and it is for that reason that we are unable to derive Cantor's paradox in $\mathrm{T}_{\lambda}^{*}$.

\section{Appendix A: Standard Second-Order Logic with Nominalized Predicates and the systems $\lambda$ HST $^{*}$ and $\lambda \mathrm{T}^{*}$}

We shall identify the different types of meaningful expressions by associating them with different natural numbers We take 0 to represent the type of all singular terms, 1 the type of all well-formed formulas (wffs), by which we mean the different forms of assertions, and $n+1$, for $n>0$, to represent the type of all $n$-place predicate expressions. We assume a denumerable infinity of individual 
variables and for each natural number $n$, a denumerable infinity of $n$-place predicate variables. (Propositional variables arc identified with 0-place predicate variables). We use ' $x$ ', ' $y$ ', ' $z$ ', with or without numerical subscripts, to refer (in the metalanguage) to individual variables, and ' $F^{n}$ ', ' $G^{n}$, , ' $R^{n}$ ' to refer to replace predicate variables. (We usually drop the superscript when the context makes clear the degree of a predicate variable, and we use ' $R$ ' to only refer to relational variables). We take $\rightarrow, \neg,=, \forall$, and $\lambda$ as primitive logical constants, and we assume that other logical constants (e.g., $\wedge, \longleftrightarrow, \exists$ ) to be defined in the usual way (as abbreviations in the metalanguage). For each natural number $n$ we recursively define the meaningful expressions of type $n$, in symbols, $\mathrm{ME}_{n}$, as follows:

1. every individual variable or constant is in $\mathrm{ME}_{0}$, and every $n$-place predicate variable or constant is in both $\mathrm{ME}_{n+1}$ and $\mathrm{ME}_{0}$;

2. if $a, b \in \mathrm{ME}_{0}$, then $(a=b) \in \mathrm{ME}_{1}$;

3. if $\pi \in \mathrm{ME}_{n+1}$, and $a_{1}, \quad a_{n}, \in \mathrm{ME}_{0}$, then $\pi\left(a_{1}, \ldots, a_{n}\right) \in \mathrm{ME}_{1}$;

4. if $\varphi \in \mathrm{ME}_{1}$, and $x_{1}, \ldots, x_{n}$, are pairwise distinct individual variables, then $\left[\lambda x_{1} \ldots x_{n} \varphi\right] \in \mathrm{ME}_{n+1}$

5. if $\varphi \in \mathrm{ME}_{1}$, then $\neg \varphi \in \mathrm{ME}_{1}$;

6. if $\varphi, \psi \in \mathrm{ME}_{1}$, then $(\varphi \rightarrow \psi) \in \mathrm{ME}_{1}$;

7. if $\varphi \in \mathrm{ME}_{1}$, and $a$ is an individual or a predicate variable, then $\forall a \varphi \in$ $\mathrm{ME}_{1}$;

8. if $\varphi \in \mathrm{ME}_{1}$, then $[\lambda \varphi] \in \mathrm{ME}_{0}$; and

9. if $n>1$, then $\mathrm{ME}_{n} \in \mathrm{ME}_{0}$.

By a singular term we mean any member of $\mathrm{ME}_{0}$, and by an $n$-place predicate expression we mean any member of $\mathrm{ME}_{n+1}$. We take the wffs to be the members of $\mathrm{ME}_{1}$. Although, by clause (9), every predicate expression is nominalizable into a singular term, not every wff is a term. We differ in this regard from what Frege would allow, but the difference is unimportant because, by clause (4), $[\lambda \varphi]$ is a wff (where $n$ is 0 ), and, by clause $(8),[\lambda \varphi]$ is a singular term. (We read $[\lambda \varphi]$ as 'that $\varphi$ ' when it occurs as a singular term.)

The extension of standard second-order logic to wffs as defined above, i.e., to wffs that may contain nominalized predicates as abstract singular terms, can be axiomatically described as follows. We use a ${ }^{~} *$ ' to indicate that the axiom applies to wffs with or without nominalized predicates, and we assume bondage and freedom and proper substitution to be defined in the usual way. We also assume modus ponens and universal generalization of individual and predicate variables to be primitive rules of inference.

$\left(\mathbf{A 0}{ }^{*}\right)$ All tautologous wffs. 
(A1*) $\forall u(\varphi \rightarrow \psi), \quad$ where $u$ is an individual or predicate variable.

$\left(\mathbf{A} 2^{*}\right) \varphi \rightarrow \forall u \varphi, \quad$ where $u$ is an individual or predicate variable not occurring free in $\varphi$.

$\left(\mathbf{A 3}^{*}\right) \exists x(a=x), \quad$ where $a$ is a singular term in which $x$ has no free occurrences.

$\left(\mathbf{L L}^{*}\right)(a=b) \rightarrow(\varphi \longleftrightarrow \psi), \quad$ where $a, b$ are singular terms, $\varphi, \psi$ are wffs, and $\psi$ comes from $\varphi$ by replacing one or more free occurrences of $b$ by free occurrences of $a .^{22}$

$\left(\lambda\right.$-Conv*) $\left[\lambda x_{1}, \ldots, x_{n} \varphi\right]\left(a_{1}, \ldots, a_{n}\right) \longleftrightarrow \varphi\left(a_{1} / x_{1}, \ldots, a_{n} / x_{n}\right), \quad$ where $a_{1}, \ldots, a_{n}$ are singular terms and each $a_{i}$ is free for $x_{i}$ in $\varphi$.

$\left(\mathbf{I D}_{\lambda}^{*}\right)\left[\lambda x_{1}, \ldots, x_{n} R\left(x_{1}, \ldots, x_{n}\right)\right]=R, \quad$ where $R$ is an $n$-place predicate variable or constant.

(Rw*) $\left[\lambda x_{1}, \ldots, x_{n} \varphi\right]=\left[\lambda y_{1}, \ldots, y_{n} \varphi\left(y_{1} / x_{1}, \ldots, y_{n} / x_{n}\right)\right], \quad$ where no $y_{i}$ occurs in $\varphi$.

$\left(\mathbf{C P}_{\lambda}^{*}\right) \exists F^{n}\left(F=\left[\lambda x_{1}, \ldots, x_{n} \varphi\right]\right), \quad$ where $F$ does not occur free in $\varphi$.

As explained in section 2, this extension of standard second-order logic leads to both Cantor's theorem and its negation, i.e., to a contradiction. This contradiction is avoided in the systems $\lambda \mathrm{HST}^{*}$ and $\lambda \mathrm{T}^{*}$ by placing a restriction on which $\lambda$-abstract are allowed to be meaningful. i.e. well-formed. Thus, as indicated in section 4 , a wff is a meaningful wff of $\lambda \mathrm{HST}^{*}$ if, and only if, every $\lambda$-abstract that occurs in that wff is homogeneously stratified (where homogeneous stratification is as defined in section 4); and a wff is a meaningful wff of $\lambda \mathrm{T}^{*}$, if, and only if, the identity sign does not occur in any $\lambda$-abstract occurring in that wff. These restrictions amount of modifying the comprehension principle $\left(\mathrm{CP}_{\lambda}^{*}\right)$, replacing it in $\lambda \mathrm{HST}^{*}$ by the homogeneously stratified comprehension principle $\left(\mathrm{HSCP}_{\lambda}^{*}\right)$, and by the comprehension principle $\left(\mathrm{CP}_{\lambda}^{* *}\right)$ in $\lambda \mathrm{T}^{*}$.

\section{Appendix B: the Systems $\mathbf{H S T}_{\lambda}^{*}$, and $\mathbf{T}_{\lambda}^{*}$}

Instead of placing any restrictions on wffs and modifying $\left(\mathrm{CP}_{\lambda}^{*}\right)$, the systems $\mathrm{HST}_{\lambda}^{*}$ and $\mathrm{T}_{\lambda}^{*}$ (as described in sections 5 and 7 , respectively) avoid the contradiction by rejecting the assumption that every concept has a concept-correlate. This is done by replacing $\left(\mathrm{A} 3^{*}\right)$ and $\left(\lambda\right.$-Conv $\left.{ }^{*}\right)$ by their "free logic" counterparts.

$\left(\mathbf{A 3}^{* *}\right) \forall x \exists y(x=y)$.

$\left(\exists / \lambda\right.$-Conv*) $\left[\lambda x_{1}, \ldots, x_{n} \varphi\right]\left(a_{1}, \ldots, a_{n}\right) \longleftrightarrow \exists x_{1} \ldots \exists x_{n}\left(a_{1}=x_{n} \wedge \ldots \wedge a_{n}=x_{n} \wedge\right.$ $\varphi), \quad$ where no $x_{i}$ is free in any $a_{j}$, for $1 \leq i, j \leq n$.

\footnotetext{
${ }^{22}$ See footnote 5 for a description of how $\left(\mathrm{LL}^{*}\right)$ applies to nominalized predicates as abstract singular terms.
} 
and adding the law of identity $(a=a)$ for all singular terms $a$, which without $\left(\mathrm{A} 3^{*}\right)$ is no longer derivable.

With these changes the principle of universal instantiation of a singular term for an individual variable, $\left(\mathrm{UI}_{1}^{*}\right)$, is no longer derivable (the way it is in $\lambda \mathrm{HST}^{*}$ and $\lambda \mathrm{T}^{*}$ except in the following qualified form:

$$
\exists y(a=y) \rightarrow[\forall x \varphi \rightarrow \varphi(a / x)] .
$$

where $a$ is any singular term that is free for $x$ in $\varphi$ and in which $y$ has no free occurrences. Without $\left(\mathrm{A} 3^{*}\right)$, the thesis that every concept has a concept-correlate, (Plato*), is no longer derivable. In place of (Plato*'), $\operatorname{HST}_{\lambda}^{*}$ has $\left(\exists / \mathrm{HSCP}_{\lambda}^{*}\right)$ (as described in section 5) as the first-order counterpart of the restricted comprehension principle ( $\left.\mathrm{HSCP}_{\lambda}^{*}\right)$, and $\mathrm{T}_{\lambda}^{*}$ has $\left(\exists / \mathrm{CP}_{\lambda}^{* *}\right)$ (as described in section 7 ) as the first-order counterpart of the restricted comprehension principle $\left(\mathrm{CP}_{\lambda}^{* *}\right)$. Otherwise, both $\mathrm{HST}_{\lambda}^{*}$ and $\mathrm{T}_{\lambda}^{*}$ have the original unrestricted comprehension principle $\left(\mathrm{CP}_{\lambda}^{*}\right)$ for concepts in general.

\section{References}

[1] Boolos, G. 1963 "The iterative conception of set", Journal of Philosophy, 68, 215-231.

[2] Cocchiarella, N.B. 1986 Logical Investigations of Predication Theory and the Problem of Universals, Naples, Bibliopolis Press.

[3] _______, 1987 Logical Studies in Early Analytic Philosophy, Columbus, Ohio State University Press.

[4] _ $\overline{8}, \overline{15}-34$.

[5] Frege, G. 1952 Translations From the Philosophical Writings of Gottlob Frege, ed. and trans. by P, Geach and M. Black, Oxford, Blackwell.

[6] Furth, Berkeley and Los Angeles, Unuversity of California Pres.

[7] Kaulbach, Oxford, Blackwell.

[8] 1980 Philosophical and Mathematical Correspondence, ed. by G. Gabriel and others, Chicago, Chicago University Press.

[9] Quine W. V. 1963 Set Theory and its Logic, Cambridge. Mass., Harvard University Press).

[10] Russell. R. 1903 The Principles of Mathematics, New York, Norton [second edition1937]. 